\title{
Incidence and genetic outcome of fetal choroid plexus cyst diagnosed at 18-23 weeks scan: review of case series
}

\author{
Olufemi Adebari Oloyede \\ Correspondence: Dr Olufemi Adebari Oloyede, Fetal and Maternal Medicine Unit, \\ Department of Obstetrics and Gynaecology, Olabisi Onabanjo University Teaching Hospital, \\ Ogun State, Nigeria; Email - oloyedeoao@gmail.com
}

Distributed under Attribution - NonCommercial - Share Alike 4.0 International (CC BY-NC-SA 4.0)

\begin{abstract}
Objectives: The objective of the study is to determine the incidence, clinical and ultrasound characteristics of choroid plexus cyst diagnosed during the $18-23$ weeks anomaly scan and the genetic outcome in 6 pregnancies that underwent molecular diagnosis. Methods: The information obtained from the clinical records of pregnancies with ultrasound diagnosis of choroid plexus cyst Data are analyzed and presented in simple frequency tables. Results: Choroid plexus cyst was diagnosed in $8(0.9 \%)$ pregnancies. The majority of the cysts were solitary $(75.0 \%)$, unilateral in location $(62.5 \%)$ and with no associated aneuploidy marker $(75.0 \%)$. Echogenic bowel and ventricular septal defect was found in 1 fetus each (25\%). Six (75.0\%) out of the 8 fetuses with chorionic plexus cyst had molecular diagnosis. Two (25\%) were trisomy 21 and 1 was trisomy 18 . Maternal age $>35$ years, multiple cysts and bilateral cyst location was reported in $2(66.7 \%)$ of the 3 fetuses with aneuploidy. Conclusions: Trisomy 21 was the more frequent aneuploidy in fetuses with choroid plexus cyst. Maternal age $>35$ years, bilateral location, multiple cysts and presence of soft aneuploidy marker are suggested as indications for molecular diagnosis in CPC.
\end{abstract}

Keywords: Fetal choroid plexus cyst, second trimester anomaly scan, invasive procedure, karyotyping, aneuploidy.

The fetal choroid plexus is a spongy villi-filled lobulation, lined by pseudostratified epithelial cells and blood vessels on the medial wall of the lateral ventricles ${ }^{1}$. The choroid plexus cyst (CPC) results from the accumulation of cerebrospinal fluid (CSF) within the plexus after 12 weeks of gestation and usually regresses by $32^{\text {nd }}$ weeks of gestation ${ }^{2}$. The sonographic description of fetal choroid plexus cyst was first reported in literature in $1984^{3}$. It is commonly diagnosed during routine $18-23$ weeks scan, with reported incidence of $0.18-3.6 \%^{4-6}$.
The clinical significance of CPC is because of its association with aneuploidy, especially trisomy 18 and trisomy $21^{3,7}$. The different scientific opinion on the role of invasive testing and karyotyping is due to differences in the interpretation of the influence of size, number and location of cyst, associated soft aneuploidy markers, maternal serum biochemistry and maternal age ${ }^{8,9}$. The aim of this study is to determine the incidence, clinical and ultrasound characteristics of CPC in fetuses of women undergoing the 18-23 weeks anomaly scan and

Received: $24^{\text {th }}$ April 2019. Accepted: $5^{\text {th }}$ June 2019 .

Oloyede OA. Incidence and genetic outcome of fetal choroid plexus cyst diagnosed at 18-23 weeks scan: review of case series. The New Indian Journal of OBGYN. 2019; 6(1): 53-6. 
their genetic outcome.

\section{Methods}

The clinical records of pregnant women that had 18-23 weeks anomaly scan from $1^{\text {st }}$ January 2015 to $31^{\text {st }}$ December 2017 were studied. The maternal age and history of aneuploidy in past children was obtained. Gestational age was calculated from an early trimester ultrasound scan or the last menstrual period, in women whose past 3 menstrual cycles were regular. Routine second trimester anomaly scan was carried out on all the women using standard check list and any fetus shown to have CPC (Figure 1) or any other obvious anomaly were rescanned. The CPC was further evaluated to determine

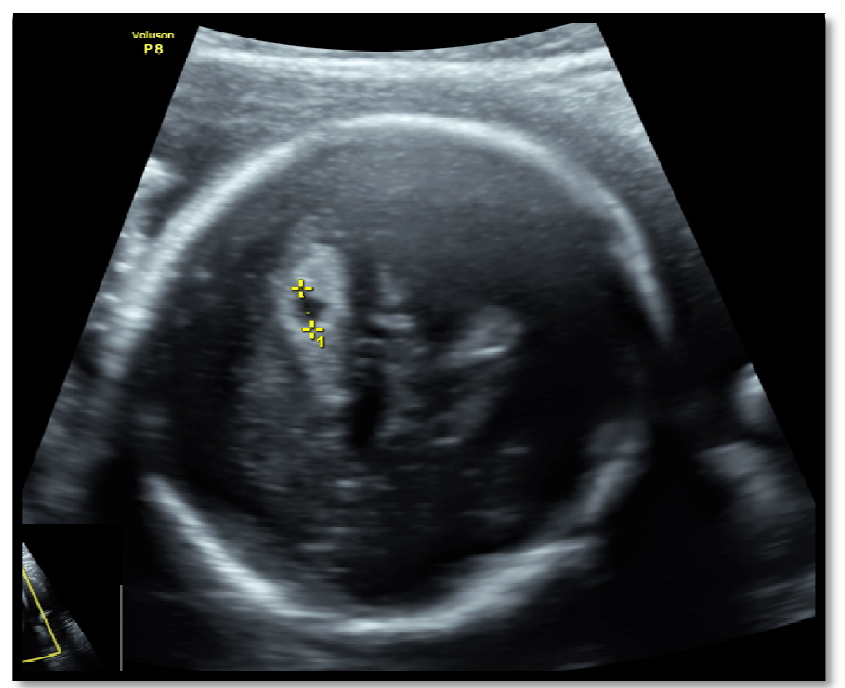

Figure 1: Unilateral Choroid Plexus Cyst at 18-23 Weeks Anomaly Scan

the size, number, location, and presence of soft markers for aneuploidy.

Ultrasound scan was performed by Fetal Medicine Foundation, London certified expert in anomaly scan, using Voluson P8 on $5.0 \mathrm{MHz}$ sector probe. Choroid plexus cysts, was defined as round or oval echo-free areas $>2 \mathrm{~mm}$ in greatest diameter that is located within the choroid plexus mass ${ }^{5}$. All the women signed informed consent for invasive procedure for molecular diagnosis and the laboratory results of all the pregnancies that had invasive procedures was retrieved from the clinical notes. Non-consenting women had postnatal molecular diagnosis done from the baby's blood.

Ethical approval for the study was obtained from the clinical practice board of High Rocks Fetal Medicine and
Genetic Diagnosis Centre, where the invasive procedures were performed. The relevant data and measurements were entered into the computer, analyzed and presented as simple frequency tables.

\section{Results}

Choroid plexus cyst was diagnosed in 8 fetuses out of the 821 women that had the 18-23 weeks anomaly scan

Table 1: Clinical and Ultrasound Scan Profile in Fetuses with Chorionic Plexus Cyst

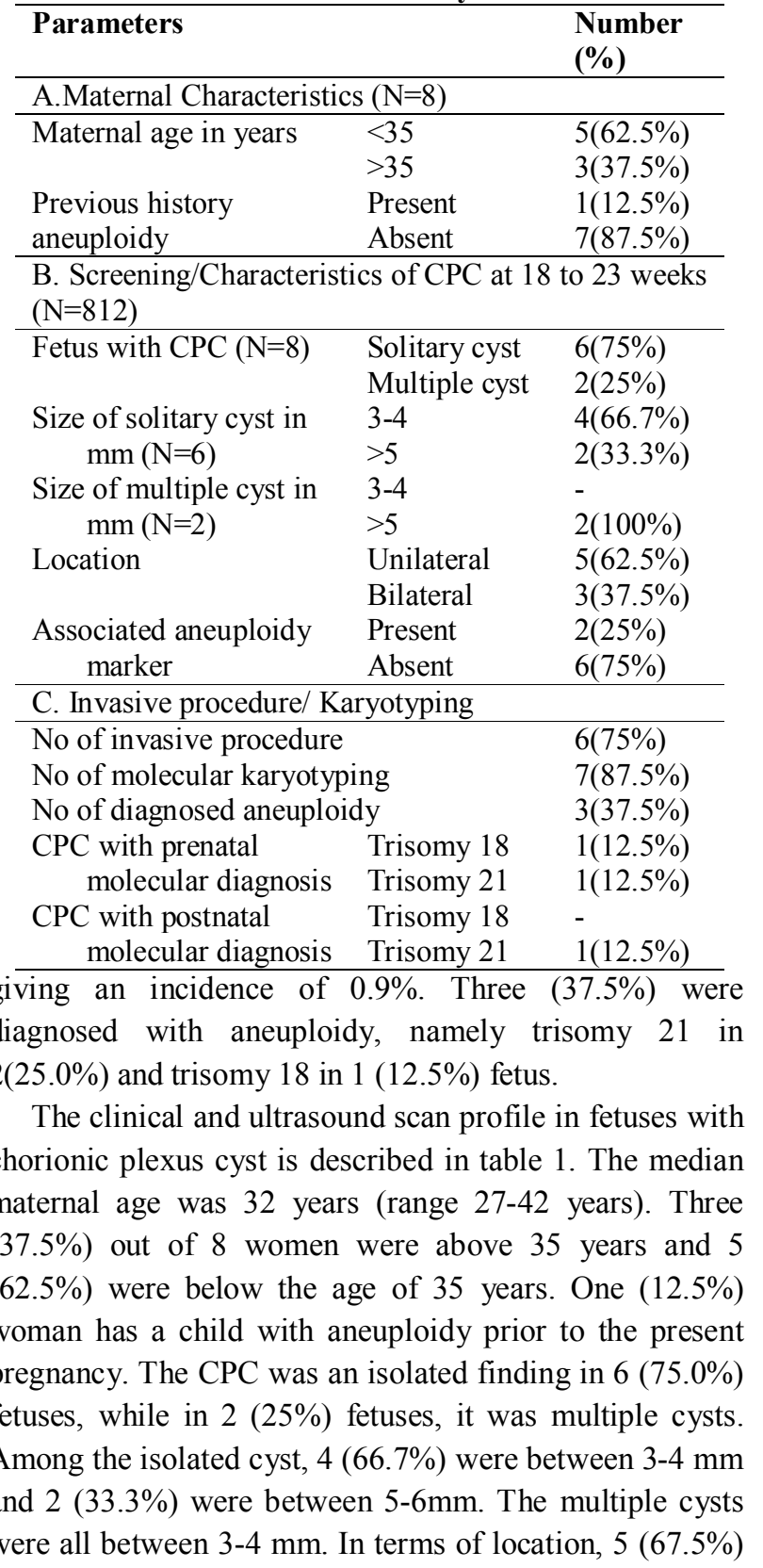


Table 2: Maternal and Ultrasound Profile in Fetuses with Aneuploidy

\begin{tabular}{llcl}
\hline Characteristics & Case 1 & $\begin{array}{c}\text { Aneuploidy cases } \\
\text { Case 2 }\end{array}$ & Case 3 \\
\hline Genetic diagnosis (Trisomy) & 21 & 18 & 21 \\
Maternal age in years & 34 & 37 & 38 \\
History of aneuploidy & Absent & Present & Absent \\
Isolated CPC & Yes & No & No \\
Associated soft markers & - & EB & VSD \\
Location & Unilateral & Bilateral & Bilateral \\
Number of cyst & Solitary & Multiple & Multiple \\
Period of diagnosis & Prenatal & Prenatal & Postnatal \\
\hline
\end{tabular}

EB: Echogenic Bowel, VSD: Ventricular Septal Defect were unilateral and $3(32.5 \%)$ bilateral. The associated soft markers were echogenic bowel and ventricular septal defect in 1 fetus each (12.5\%). Out of the 8 pregnancies with diagnosis of fetal choroid plexus cysts, $6(75.0 \%)$ consented to invasive procedure and $2(25.0 \%)$ declined.

Three $(37.5 \%)$ fetuses out of 8 diagnosed with CPC had prenatal molecular confirmation of aneuploidy, namely trisomy 21 and 18, while the third had postnatal molecular diagnosis of trisomy 21.

Table 2 presents the analysis of maternal and ultrasound scan characteristics in 3 fetuses that were confirmed to be trisomy. Trisomy 21 was diagnosed in 2 $(66.7 \%)$ fetuses and trisomy 18 in $1(33.3 \%)$ fetus. In 2 $(66.7 \%)$ fetuses, the maternal age was $>35$ years, aneuploidy markers were present, and cysts were multiple. History of a previous child with aneuploidy was obtained in only $1(33.3 \%)$ woman.

The first fetus with trisomy 21 was diagnosed in a 34 year old woman, without a previous history of aneuploidy. The CPC was solitary, unilateral and without any associated soft marker. Trisomy 18 was diagnosed in a fetus with multiple CPC, bilateral and has echogenic bowel. The mother was 37 years and has a child with trisomy 21 . The $3^{\text {rd }}$ fetus was diagnosed in a woman that declined prenatal molecular testing. The fetus has multiple, bilateral CPC and presence of VSD. Post natal clinical suspicion of trisomy 21 was confirmed through molecular karyotyping and the ventricular septal defect was successfully repaired. The second non-consenting woman was diagnosed with bilateral multiple CPC, but without soft marker present in the fetus was lost to follow up.

\section{Discussion}

The incidence of choroid plexus cyst in the study was $0.9 \%$, while trisomy 21 was the more frequently diagnosed aneuploidy condition in the 6 reviewed cases. This incidence of CPC reported by various studies was of 0.18-3.6\% 4, 5, 6 . The wide difference in the incidence may be due to the fact that the cyst usually appear only transiently in early pregnancy and tend to disappear as pregnancy advances in gestational age ${ }^{6}$. The molecular laboratory outcome was similar to the report from other studies that show that trisomy 18 and 21 were the frequently associated aneuploidy, with preponderance of trisomy 18 over $21^{4}$. In the contrary however, this study shows more of trisomy 21 over 18. It is possible to explain this study distribution to the proven lethality of trisomy 18 , which results in the death of more of affected fetuses in utero compared with fetuses affected by trisomy 21 . Thirty percent of trisomy dies between 12 weeks and delivery, while $80 \%$ of trisomy 18 dies between 12 weeks and delivery ${ }^{10}$. Twothirds of trisomy 18 detected in the second trimester will spontaneously die in utero and of the live born, 90-95\% will die before end of their first year birthday 10 . Consequently, it is more likely for a surviving fetus with CPC to be diagnosed as trisomy 21. It may however be explained as chance event rather than the true proportion that would be obtained in a larger series. The study shows that maternal age do not have any effect on the incidence of CPC, but a significant effect may be on the occurrence of aneuploidy.

The management in pregnancies with diagnosis of CPC is either 'watchful expectancy' or counseling for invasive testing and karyotyping, with bias in favor karyotyping because of the significant association between CPC and aneuploidy ${ }^{8}$. In many centers, isolated CPC without associated soft markers is an acceptable indication for karyotyping, while for some researcher, it is not a routine indication for karyotyping ${ }^{3,4,9}$. There is also no consensus about the strength of isolated CPC as an 
The New Indian Journal of OBGYN. 2019 (July-December); 6(1)

indication for trisomy 18 and 21 . While some researchers believe that association of CPC with trisomy 21 is not as strong as with trisomy 18 , other views are not in agreement 5,6 . Irrespective of scientific opinion, it is generally agreed that karyotyping carried out in early pregnancy provides opportunity for women to confirm a suspected diagnosis and to make informed decision about the future of the pregnancy.

The concern about procedure related risk of abortion that is associated with invasive prenatal testing could be a major factor in counseling for molecular diagnosis. This can however be avoided with the introduction of non invasive prenatal testing ${ }^{11}$.

\section{Conclusion}

In this study, CPC was more frequently associated with trisomy 21 than trisomy 18 . Maternal age $>35$ years, presence of soft aneuploidy marker and multiple and bilateral CPC, may suggest higher likelihood for trisomy 21 in the fetus and should be considered as an indication for karyotyping. This study was to describe the observations on the associations between CPC and aneuploidy. A larger series will improve on the strength of its scientific inferences.

\section{Acknowledgement}

We would like to appreciate the medical record staff for record retrieval data extraction and analysis.

\section{Conflict of interest: None. Disclaimer: Nil.}

\section{References}

1.Pappalardo EM, Militello M, Rapisarda G, Imbruglia L, Recupero S, Ermito S, et al. Fetal intracranial cysts: prenatal diagnosis and outcome. Journal of prenatal medicine. 2009: 3 (2): $28-30$.

2.Jeon JH, Lee SW, Ko JK, Choi BG, Cha SH, Song GS, et al. Neuroendoscopic removal of large choroid plexus cyst: a case report. J Korean Med Sci. 2005: 20 (2): 335-9.
3.Chudleigh P, Pearce JM, Campbell S. The prenatal diagnosis of transient cysts of the fetal choroid plexus. Prenat Diagn. 1984; 4: 135-7.

4. Tayyar AT, Tayyar M. Multiple Choroid Plexus Cysts: a case report. Medicine Science Intenational Journal. 2016; 5(4):1019-20

5.Geary MP, Lamont R. Isolated Choroid Plexus cysts and with fetal aneuploidy in an unselected population.

Ultrasound in Obstetrics \& Gynecology. 1997; 10: 171-3.

6.Rimoin D, Pyeritz R, Korf B, eds. Emery and Rimoin's Principles and Practice of Medical Genetics. 6th edition. Massachusetts: Academic Press; 2013.

7.Irani S, Ahmadi F, Javam M, Taghi dizaj AV, Niknejad F. Outcome of isolated fetal choroid plexus cyst detected in prenatal sonography among infertile patients referred to Royan Institute: A 3-year study. Iran Journal of Reproductive Medicine. 2015; 13(9): 571-6.

8. Walkinshaw S, Pilling D, Spriggs A. Isolated choroid plexus cysts - the need for routine offer of karyotyping. Prenatal Diagnosis. 1994; 14(8): 663 -7.

9.ACOG Practice Bulletin. Clinical management guidelines for obstetrician - gynecologists. Prenatal diagnosis of fetal chromosomal abnormalities. Obstetetrics and Gynecology. 2001; 97: 1-12

10.Nicolaides $\mathrm{KH}$, editor. The 11 to $13+6$ Weeks scan. London: Fetal Medicine Foundation; 2004.

11.American College of Obstetricians and Gynecologists Committee on Genetics. Committee opinion no. 545: noninvasive prenatal testing for fetal aneuploidy. Obstet Gynecol. 2012; 120(6):1532-4.

\section{Olufemi Adebari Oloyede ${ }^{1}$ \\ ${ }^{1}$ Fetal and Maternal Medicine Unit, Department of Obstetrics and Gynaecology, Olabisi Onabanjo University Teaching Hospital, Ogun State, Nigeria.}

